Volume 134, Number 1, Pages 207-214

S 0002-9939(05)07968-2

Article electronically published on June 2, 2005

\title{
UNIMODULAR FUNCTIONS AND INTERPOLATING BLASCHKE PRODUCTS
}

\author{
GEIR ARNE HJELLE
}

(Communicated by Juha M. Heinonen)

\begin{abstract}
The result by Bourgain that every unimodular function $\psi$ on the unit circle can be factored as $\psi=e^{i \tilde{v}} B_{1} \bar{B}_{2}$ with $B_{1}$ and $B_{2}$ Blaschke products can be improved. We show that the same result holds with $B_{1}$ and $B_{2}$ interpolating Blaschke products. This will at the same time be a refinement of Jones's result that every unimodular function can be approximated in the $H^{\infty}$-norm by a ratio of interpolating Blaschke products.
\end{abstract}

\section{Introduction}

A Blaschke product is an $H^{\infty}$-function on the open unit disk $\mathbb{D}$ of the form

$$
B(z)=z^{m} \prod_{\left|z_{n}\right| \neq 0} \frac{-z_{n}}{\left|z_{n}\right|} \frac{z-z_{n}}{1-\bar{z}_{n} z}
$$

where $\left\{z_{n}\right\}$ is a set of points in $\mathbb{D}$ such that

$$
\sum\left(1-\left|z_{n}\right|\right)<\infty
$$

and $m$ is the number of $z_{n}$ 's equal to 0 . The set $\left\{z_{n}\right\}$ is called the zero set of the Blaschke product, as the zeros of $B(z)$ are precisely the points $z_{n}$ counted with multiplicity. We have $|B(z)| \leq 1$ in $\mathbb{D}$ and non-tangential limits $|B(z)|=1$ almost everywhere on the unit circle $\mathbb{T}$. See [4, pp. 53-57] for further information on Blaschke products. The Blaschke product is called interpolating if the zero set is an interpolating sequence. That is, if every interpolation problem

$$
f\left(z_{j}\right)=a_{j}, \quad j \in \mathbb{N},\left(a_{j}\right) \in \ell^{\infty},
$$

has a solution $f \in H^{\infty}$. A famous result by Carleson shows that, equivalently, a Blaschke product with zero set $\left\{z_{n}\right\}$ is an interpolating Blaschke product if and only if the following two conditions hold [2]:

i) $\inf _{n \neq m} d\left(z_{n}, z_{m}\right)>0$.

ii) For all Carleson squares $Q=\left\{r e^{i \theta}: \theta_{0}<\theta<\theta_{0}+\ell(Q), 1-\ell(Q)<r<1\right\}$,

$$
\sum_{z_{n} \in Q}\left(1-\left|z_{n}\right|\right)<C \ell(Q)
$$

for some constant $C$.

Received by the editors April 8, 2004 and, in revised form, August 25, 2004.

2000 Mathematics Subject Classification. Primary 30D50, 30E10.

Research supported by grants from the Research Council of Norway, project \#155060, and the Norwegian University of Science and Technology. 
Here $\ell(Q)$ is the base length of $Q$, while $d$ denotes the pseudo-hyperbolic distance

$$
d(z, w)=\left|\frac{z-w}{1-\bar{w} z}\right|
$$

In 1969 Douglas and Rudin asked whether $\int_{\mathbb{T}} \log |f|>-\infty$ was both a sufficient and necessary condition on $f \not \equiv 0$ for $f$ to be of the form $f=g \bar{h}$ with $g, h \in H^{\infty}[3$. The question was answered affirmatively by Bourgain in 1986 [1]. He proved the following result, from which the answer to Douglas's and Rudin's problem follows easily.

Theorem 1. Suppose $a \in L^{\infty}(\mathbb{T})$ with $\|a\|_{\infty} \leq \pi$. Then there are Blaschke products $B_{1}$ and $B_{2}$ such that

$$
\left\|\left(a-\operatorname{Arg} \frac{B_{1}}{B_{2}}\right)^{\sim}\right\|_{\infty}<c
$$

where $c$ is a constant.

We use $f \mapsto \tilde{f}$ for the conjugation operator. By examining Bourgain's proof carefully, we may strengthen the theorem. In fact,

$$
\left\|a-\operatorname{Arg} \frac{B_{1}}{B_{2}}\right\|_{\infty}+\left\|\left(a-\operatorname{Arg} \frac{B_{1}}{B_{2}}\right)^{\sim}\right\|_{\infty}<\varepsilon
$$

for every $\varepsilon>0$. Define $v=-\left(a-\operatorname{Arg} \frac{B_{1}}{B_{2}}\right)^{\sim}$. Then $v \in L^{\infty}$, and because $\left|B_{j}\right|=1$ almost everywhere on $\mathbb{T}$, Theorem 1 can be reformulated as follows:

Theorem 1'. Suppose $\psi$ is a unimodular function on $\mathbb{T}$. Then for every $\varepsilon>0$ there exist Blaschke products $B_{1}$ and $B_{2}$ such that

$$
\psi=e^{i \tilde{v}} \frac{B_{1}}{B_{2}}=e^{i \tilde{v}} B_{1} \overline{B_{2}}
$$

for some $v \in L^{\infty}$ with $\|v\|_{\infty}<\varepsilon$ and $\|\tilde{v}\|_{\infty}<\varepsilon$.

The question of whether an arbitrary Blaschke product (and thus an arbitrary inner function) can be approximated by an interpolating Blaschke product has been investigated for some time. The problem, which is still open, was posed by Peter Jones who in 1981 approximated an arbitrary unimodular function by a ratio of interpolating Blaschke products in the $H^{\infty}$-norm [6]. In the present paper we do something similar. We approximate a ratio of Blaschke products $B_{1} / B_{2}$ by a ratio of interpolating Blaschke products $B_{1}^{*} / B_{2}^{*}$ in the sense that

$$
\frac{B_{1}}{B_{2}}=e^{i \tilde{v}} \frac{B_{1}^{*}}{B_{2}^{*}},
$$

with $v \in L^{\infty}$ and where $\|v\|_{\infty}$ and $\|\tilde{v}\|_{\infty}$ are small. By doing so, we show that Bourgain's result also holds for interpolating Blaschke products.

Theorem 2. Suppose $\psi$ is a unimodular function on $\mathbb{T}$. Then for every $\varepsilon>0$ there exist interpolating Blaschke products $B_{1}^{*}$ and $B_{2}^{*}$ such that

$$
\psi=e^{i \tilde{v}} \frac{B_{1}^{*}}{B_{2}^{*}}=e^{i \tilde{v}} B_{1}^{*} \overline{B_{2}^{*}}
$$

for some $v \in L^{\infty}$ with $\|v\|_{\infty}<\varepsilon$ and $\|\tilde{v}\|_{\infty}<\varepsilon$.

This result can also be viewed as a strengthened version of Jones's theorem. 


\section{UNIMODULAR FUNCTIONS ON $\mathbb{T}$}

To prove Theorem 2 we use a result of Marshall and Stray [7] concerning the product of interpolating Blaschke products, and a result of Garnett and Nicolau [5] showing how a Blaschke product can be approximated by a ratio of interpolating Blaschke products.

Lemma 3. Let $B_{1}^{*}$ and $B_{2}^{*}$ be interpolating Blaschke products. Then for every $\varepsilon>0$ there is an interpolating Blaschke product, $B^{*}$, such that

$$
B_{1}^{*} B_{2}^{*}=B^{*} e^{i \tilde{v}}
$$

on $\mathbb{T}$ for some $v \in L^{\infty}$ with $\|v\|_{\infty}<\varepsilon$ and $\|\tilde{v}\|_{\infty}<\varepsilon$.

Proof. This lemma can, although not stated explicitly, be inferred from [7]. The main part of the argument can also be found in [8, pp. 101-103], so we only sketch it here. Denote the zero sets of $B_{1}^{*}$ and $B_{2}^{*}$ by $\left\{a_{n}\right\}$ and $\left\{b_{n}\right\}$ respectively. Choose $\delta$ so small that

$$
d\left(a_{n}, a_{m}\right) \geq 2 \delta \quad \text { and } \quad d\left(b_{n}, b_{m}\right) \geq 2 \delta \quad \text { for all } n \neq m .
$$

By moving zeros from $B_{1}^{*}$ to $B_{2}^{*}$ if necessary, the closed disks

$$
\Delta_{j}=\left\{z: d\left(z, a_{j}\right) \leq c \delta\right\}, \quad c \text { small, }
$$

are disjoint and contain exactly one $b_{n}$ each. See Figure1. We must show that also the zeros left in $B_{1}^{*}$ can be separated from those of $B_{2}^{*}$ such that condition i) holds. By Frostman's theorem [4, p. 79] there is an $\varepsilon_{0} \in\left(c \delta, \frac{4}{3} c \delta\right)$ such that

$$
\widehat{B}_{1}^{*}(z)=\frac{B_{1}^{*}(z)-\varepsilon_{0}}{1-\varepsilon_{0} B_{1}^{*}(z)}
$$

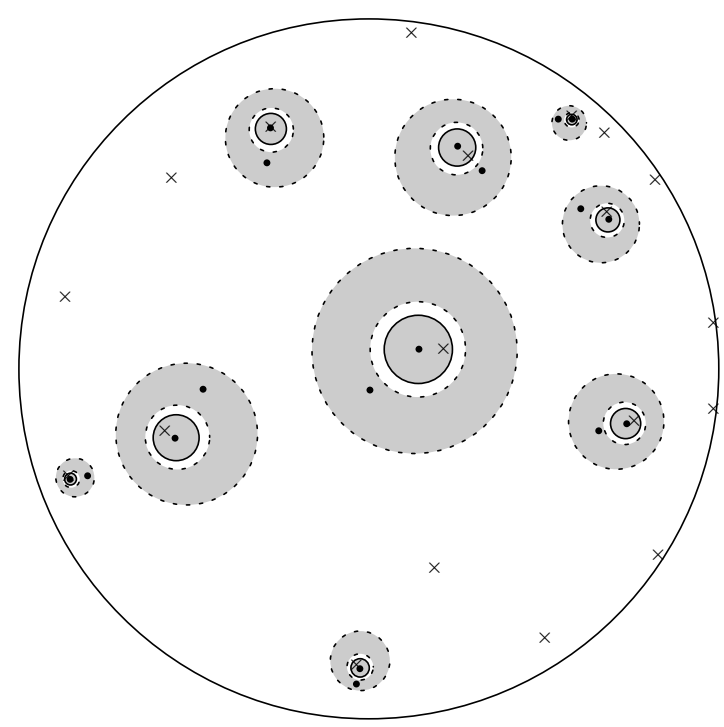

Figure 1 . The closed disks $\Delta_{j}$ and the corresponding pseudohyperbolic annuli $A_{j}$. The zeros of $B_{1}^{*}$ and $\widehat{B}_{1}^{*}$ are marked $\bullet$, while the zeros of $B_{2}^{*}$ are marked $\times$. 
is a Blaschke product. It can then be shown that the zeros of $\widehat{B}_{1}^{*}$ lie in the pseudohyperbolic annuli

$$
A_{j}=\left\{z: \varepsilon_{0}<d\left(z, a_{j}\right)<\delta\right\} .
$$

Thus they are separated from the zeros of $B_{2}^{*}$. It follows that $B^{*}=\widehat{B}_{1}^{*} B_{2}^{*}$ is an interpolating Blaschke product approximating $B_{1}^{*} B_{2}^{*}$. On $\mathbb{T}, B_{1}^{*} \overline{B_{1}^{*}}=\left|B_{1}^{*}\right|^{2}=1$ almost everywhere. Hence we can write

$$
\widehat{B}_{1}^{*}=\frac{B_{1}^{*}-\varepsilon_{0}}{1-\varepsilon_{0} B_{1}^{*}}=\frac{B_{1}^{*}\left(1-\varepsilon_{0} \overline{B_{1}^{*}}\right)}{1-\varepsilon_{0} B_{1}^{*}}=B_{1}^{*} \frac{\bar{h}}{h}
$$

where $h=1-\varepsilon_{0} B_{1}^{*}$ is an outer function. So $h=e^{u+i \tilde{u}}$ with $u \in L^{\infty}$. This gives $\widehat{B}_{1}^{*}=e^{-2 i \tilde{u}} B_{1}^{*}$ and by taking $\varepsilon_{0}$ small enough, we can ensure that $\|u\|_{\infty}$ and $\|\tilde{u}\|_{\infty}$ are less than $\frac{\varepsilon}{2}$.

Observe that this lemma is easily extended to finite products of interpolating Blaschke products.

The main step in the proof of Theorem 2 is the approximation of Blaschke products by ratios of interpolating Blaschke products. This is accomplished through the following lemma, which is a slight modification of a result by Garnett and Nicolau [5].

Lemma 4. Let $B$ be a Blaschke product. Then for every $\varepsilon>0$ there exist interpolating Blaschke products $B_{1}^{*}$ and $B_{2}^{*}$ such that

$$
B=e^{i \tilde{v}} \frac{B_{1}^{*}}{B_{2}^{*}}=e^{i \tilde{v}} B_{1}^{*} \overline{B_{2}^{*}}
$$

on $\mathbb{T}$ for some $v \in L^{\infty}$ with $\|v\|_{\infty}<\varepsilon$ and $\|\tilde{v}\|_{\infty}<\varepsilon$.

Proof. We follow the same construction as Garnett and Nicolau. Let $0<\alpha<\beta<1$, $M=2^{K}>1$ and $\delta<1$ be constants whose values will be determined later. Note that by applying a preliminary conformal mapping we may assume $|B(0)|>\beta$. We will consider dyadic Carleson squares of the form

$$
Q_{n, j}=\left\{r e^{i \theta}: 2 \pi j 2^{-n} \leq \theta<2 \pi(j+1) 2^{-n}, 1-2^{-n} \leq r<1\right\}
$$

and their top-halves $T\left(Q_{n, j}\right)$. Let $G_{1}=\left\{Q_{1}^{(1)}, Q_{2}^{(1)}, \ldots\right\}$ be the set of maximal $Q_{n, j}$ with

$$
\inf _{T\left(Q_{n, j}\right)}|B(z)|<\alpha .
$$

Write $S_{p, k}^{(1)}, 1 \leq p \leq M=2^{K}$, for the $M$ different $Q_{n+K, j} \subset Q_{n, j}=Q_{k}^{(1)}$, and let $H_{1}=\left\{V_{1}^{(1)}, V_{2}^{(1)}, \ldots\right\}$ be the set of maximal $Q_{n, j}$ for which $V_{l}^{(1)} \subset Q_{k}^{(1)}$ for some $Q_{k}^{(1)}$ and

$$
\inf _{T\left(V_{l}^{(1)}\right)}|B(z)|>\beta .
$$

The function $|B|$ has non-tangential limit 1 almost everywhere, so

$$
\sum_{V_{l}^{(1)} \subset Q_{k}^{(1)}} \ell\left(V_{l}^{(1)}\right)=\ell\left(Q_{k}^{(1)}\right) .
$$

Let

$$
f(z)=\frac{B(z)-w_{0}}{1-\overline{w_{0}} B(z)}
$$


with $w_{0}=B\left(z_{0}\right), z_{0} \in Q_{k}^{(1)}$ and $\left|w_{0}\right|=\alpha$. If $1-\beta$ is small, then Schwarz's Lemma applied to $f$ implies that

$$
\sup _{T\left(S_{p, k}^{(1)}\right)}|B(z)|<\beta .
$$

We may also deduce that $V_{l}^{(1)} \subset S_{p, k}^{(1)}$ for some $p, k$.

Next we iterate the construction. Let $G_{2}=\left\{Q_{1}^{(2)}, Q_{2}^{(2)}, \ldots\right\}$ be the set of maximal $Q_{n, j}$ such that

$$
Q_{n, j} \subset V_{l}^{(1)} \in H_{1} \quad \text { and } \quad \inf _{T\left(Q_{n, j}\right)}|B(z)|<\alpha .
$$

The $Q_{k}^{(2)}$ are relatively few. In fact, by [4, p. 334], given $\varepsilon_{0}>0$ we can take $(1-\beta) /(1-\alpha)$ so small that

$$
\sum_{Q_{k}^{(2)} \subset V_{l}^{(1)}} \ell\left(Q_{k}^{(2)}\right)<\varepsilon_{0} \ell\left(V_{l}^{(1)}\right) .
$$

The sets $\left\{S_{p, k}^{(2)}\right\}$ and $H_{2}=\left\{V_{l}^{(2)}\right\}$ are constructed in the same manner as above. By repeating the argument we obtain

$$
Q_{k}^{(m)} \supset S_{p, k}^{(m)} \supset V_{l}^{(m)} \supset Q_{k}^{(m+1)} .
$$

Define

$$
R_{p, k}^{(m)}=S_{p, k}^{(m)} \backslash \bigcup_{V_{l}^{(m)} \subset S_{p, k}^{(m)}} V_{l}^{(m)}
$$

and observe that the zeros of $B(z)$ are in

$$
\bigcup_{k, m}\left(Q_{k}^{(m)} \backslash \underset{V_{l}^{(m)} \subset Q_{k}^{(m)}}{\bigcup} V_{l}^{(m)}\right) .
$$

By taking $1-\alpha$ small we can make all zeros from $Q_{k}^{(m)} \backslash \bigcup V_{l}^{(m)}$ fall into $\bigcup_{p=1}^{M} R_{p, k}^{(m)}$.

Factor $B$ as $B=B_{1} \cdots B_{M}$ where $B_{p}$ has zeros only in $\bigcup_{k, m} R_{p, k}^{(m)}$. Fix $p$ and set

$$
\Gamma_{p, k}^{(m)}=\partial R_{p, k}^{(m)} \backslash \partial S_{p, k}^{(m)}
$$

See Figure 2] Mark points $z_{\nu}^{*}=z_{\nu}^{*}(k, m, p)$ on $\Gamma_{p, k}^{(m)}$ such that

$$
d\left(z_{\nu}^{*}, z_{\nu+1}^{*}\right)=\delta .
$$

Let $B_{p}^{*}$ be the Blaschke product with zeros $\bigcup_{k, m} z_{\nu}^{*}(k, m, p)$. Then condition i) holds by (4). From the definition of the $z_{\nu}^{*}$ 's there is a constant $c$ dependent on $\delta$ such that

$$
\sum_{z_{\nu}^{*} \in Q_{k}^{(m)}}\left(1-\left|z_{\nu}^{*}\right|\right) \leq c \sum_{V_{l}^{(n)} \subset Q_{k}^{(m)}} \ell\left(V_{k}^{(n)}\right) .
$$

Hence by (2) and (3),

$$
\sum_{z_{\nu}^{*} \in Q_{k}^{(m)}}\left(1-\left|z_{\nu}^{*}\right|\right)<\frac{c}{1-\varepsilon_{0}} \ell\left(Q_{k}^{(m)}\right),
$$

so condition ii) holds for all dyadic Carleson squares, and therefore for all Carleson squares. It follows that $B_{p}^{*}$ is an interpolating Blaschke product. By Lemma 3 there is then an interpolating Blaschke product $B^{*}=e^{i \tilde{v}} B_{1}^{*} \cdots B_{M}^{*}$ on $\mathbb{T}$. To finish 

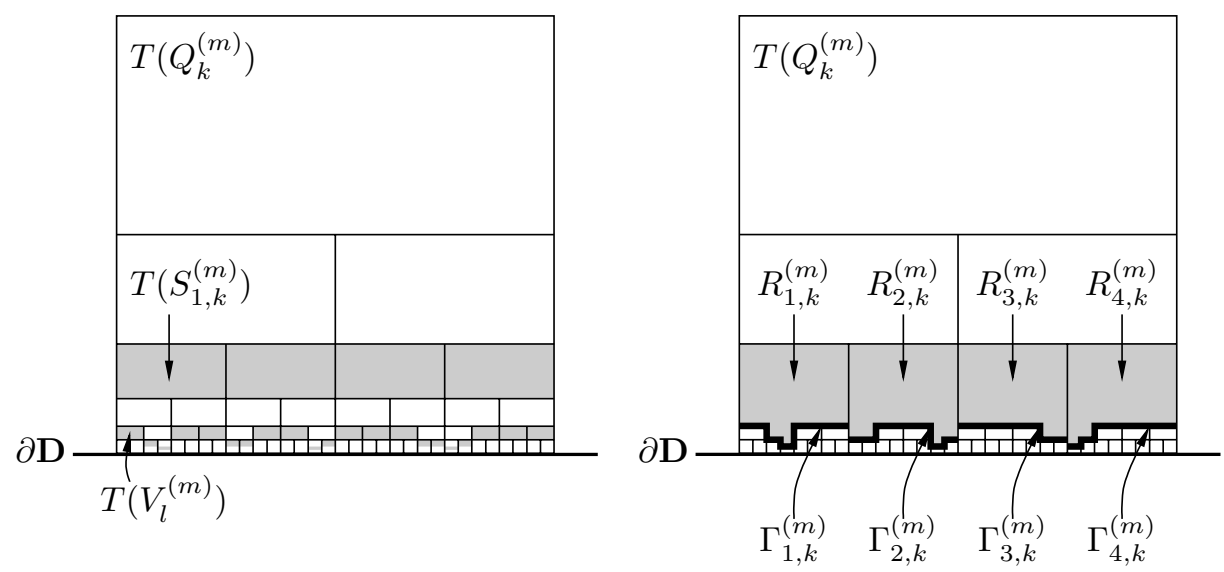

Figure 2. Example of a $Q_{k}^{(m)}$ with $S_{p, k}^{(m)}, V_{l}^{(m)}, R_{p, k}^{(m)}$ and $\Gamma_{p, k}^{(m)}$

the proof we will need to show the existence of yet another interpolating Blaschke product $C^{*}$ such that $C^{*}=e^{i \tilde{u}} B B^{*}$.

Before doing so we state three lemmas from [5] which help us with this last part.

Lemma 5. Let $B$ be a Blaschke product and let $\left\{z_{\nu}\right\}$ be its zeros, counted with their multiplicities. Then $B$ is a finite product of interpolating Blaschke products if and only if there exist positive constants $d_{0}, \delta_{0}$ such that for each $z_{\nu}$ there is $w_{\nu}$ with

$$
d\left(z_{\nu}, w_{\nu}\right) \leq d_{0}
$$

and

$$
\left(1-\left|w_{\nu}\right|^{2}\right)\left|B^{\prime}\left(w_{\nu}\right)\right| \geq \delta_{0}
$$

Lemma 6. $\left|B_{p}^{*}\right| \leq \delta^{1 / 4}$ on $\bigcup_{k, m} R_{p, k}^{(m)}$.

Lemma 7. There exist $A=A(\alpha, \beta, \delta, M)$ and $\eta=\eta(\alpha, \beta, \delta, M)>0$ so that if

$$
\inf _{\xi \in \cup_{k, m} R_{p, k}^{(m)}} d(z, \xi)>A
$$

and if

$$
\left|B_{p} B_{p}^{*}(z)\right|=\delta^{1 / 8},
$$

then

$$
\left(1-|z|^{2}\right)\left|\left(B_{p} B_{p}^{*}\right)^{\prime}(z)\right| \geq \eta .
$$

Proof of Lemma 4 continued. By Frostman's theorem there is a constant $\gamma$ with $|\gamma|=\delta^{1 / 8}$ so that

$$
C_{p}=\frac{B_{p} B_{p}^{*}-\gamma}{1-\bar{\gamma} B_{p} B_{p}^{*}}
$$

is a Blaschke product. Let $z_{0}$ be such that $C_{p}\left(z_{0}\right)=0$. Then $\left|\left(B_{p} B_{p}^{*}\right)\left(z_{0}\right)\right|=\delta^{1 / 8}$ and

$$
\left(1-\left|z_{0}\right|^{2}\right)\left|C_{p}^{\prime}\left(z_{0}\right)\right| \geq \frac{1-\left|z_{0}\right|^{2}}{1-|\gamma|^{2}}\left|\left(B_{p} B_{p}^{*}\right)^{\prime}\left(z_{0}\right)\right|
$$


If (5) holds, Lemma 7 implies that

$$
\left(1-\left|z_{0}\right|^{2}\right)\left|C_{p}^{\prime}\left(z_{0}\right)\right| \geq \frac{\eta}{1-|\gamma|^{2}}>0 .
$$

If, on the other hand, (5) does not hold, there is a $\xi \in \bigcup_{k, m} R_{p, k}^{(m)}$ with $d\left(z_{0}, \xi\right) \leq A$. From Lemma 6 we have $\left|\left(B_{p} B_{p}^{*}\right)(\xi)\right| \leq \delta^{1 / 4}$, so somewhere along the hyperbolic geodesic from $z_{0}$ to $\xi$ there is a point $w$ with

$$
\left(1-|w|^{2}\right)\left|\left(B_{p} B_{p}^{*}\right)^{\prime}(w)\right|>\hat{\eta}>0 \quad \text { and } \quad d(z, w)<A .
$$

Then also

$$
\left(1-|w|^{2}\right)\left|C_{p}^{\prime}(w)\right|>0 .
$$

So either way Lemma 5 tells us that $C_{p}$ is a finite product of interpolating Blaschke products.

Lemma 3 then gives us the existence of $u_{p} \in L^{\infty}$ such that $C_{p}^{*}=e^{-i \tilde{u}_{p}} C_{p}$ are interpolating Blaschke products on $\mathbb{T}$. Furthermore,

$$
C_{p}^{*}=\frac{B_{p} B_{p}^{*}\left(1-\gamma \overline{B_{p} B_{p}^{*}}\right)}{1-\bar{\gamma} B_{p} B_{p}^{*}} e^{-i \tilde{u}_{p}}=e^{-i \tilde{v}_{p}} B_{p} B_{p}^{*} \quad \text { or } \quad B_{p}=e^{i \tilde{v}_{p}} \frac{C_{p}^{*}}{B_{p}^{*}},
$$

and

$$
B=B_{1} \cdots B_{M}=e^{i \tilde{v}} \frac{C^{*}}{B^{*}}=e^{i \tilde{v}} C^{*} \overline{B^{*}}
$$

where $B^{*}=B_{1}^{*} \cdots B_{M}^{*}$ and $C^{*}=C_{1}^{*} \cdots C_{M}^{*}$ are interpolating Blaschke products. Also $v=v_{1}+\cdots+v_{M} \in L^{\infty}$ with $\|v\|_{\infty}<\varepsilon$ and $\|\tilde{v}\|_{\infty}<\varepsilon$.

Proof of Theorem 2. From (1) we have that $\psi=e^{i \tilde{u}} \frac{B_{1}}{B_{2}}$ for some $u \in L^{\infty}$ with $\|u\|_{\infty}<\frac{\varepsilon}{5}$ and $B_{1}$ and $B_{2}$ Blaschke products. Lemma 4 aids us in approximating $B_{1}$ and $B_{2}$ by interpolating Blaschke products $B_{i, j}^{*}$,

$$
\psi=e^{i \tilde{u}} \frac{e^{i \tilde{u}_{1}} B_{1,1}^{*} / B_{1,2}^{*}}{e^{i \tilde{u}_{2}} B_{2,1}^{*} / B_{2,2}^{*}}=e^{i\left(\tilde{u}+\tilde{u}_{1}-\tilde{u}_{2}\right)} \frac{B_{1,1}^{*} B_{2,2}^{*}}{B_{1,2}^{*} B_{2,1}^{*}} .
$$

By Lemma 3, these products may again be approximated by interpolating Blaschke products. Thus,

$$
\psi=e^{i\left(\tilde{u}+\tilde{u}_{1}-\tilde{u}_{2}\right)} \frac{B_{1}^{*} / e^{i \tilde{v}_{1}}}{B_{2}^{*} / e^{i \tilde{v}_{2}}}=e^{i\left(\tilde{u}+\tilde{u}_{1}-\tilde{u}_{2}-\tilde{v}_{1}+\tilde{v}_{2}\right)} \frac{B_{1}^{*}}{B_{2}^{*}}=e^{i \tilde{v}} B_{1}^{*} \overline{B_{2}^{*}},
$$

where $B_{1}^{*}$ and $B_{2}^{*}$ are interpolating Blaschke products and $v=u+u_{1}-u_{2}-v_{1}+v_{2} \in$ $L^{\infty}$. The norms of $u_{1}, u_{2}, v_{1}$ and $v_{2}$ can all be taken less than $\frac{\varepsilon}{5}$; so also can the norms of $\tilde{u}_{1}, \tilde{u}_{2}, \tilde{v}_{1}$ and $\tilde{v}_{2}$. Thus $\|v\|_{\infty}<\varepsilon$ and $\|\tilde{v}\|_{\infty}<\varepsilon$.

\section{ACKNOWLEDGMENT}

I am grateful to my Ph.D. advisor Kristian Seip for proposing this problem and for valuable help. 


\section{REFERENCES}

1. Jean Bourgain, A problem of Douglas and Rudin on factorization, Pacific J. Math. 121 (1986), no. 1, 47-50. MR0815031 (87d:30040)

2. Lennart Carleson, An interpolation problem for bounded analytic functions, Amer. J. Math. 80 (1958), 921-930. MR0117349 (22:8129)

3. R.G. Douglas and Walter Rudin, Approximation by inner functions, Pacific J. Math. 31 (1969), no. 2, 313-320. MR0254606 (40:7814)

4. John B. Garnett, Bounded analytic functions, Academic Press, 1981. MR0628971 (83g:30037)

5. John B. Garnett and Artur Nicolau, Interpolating Blaschke products generate $H^{\infty}$, Pacific J. Math. 173 (1996), no. 2, 501-510. MR.1394402 (97f:30050)

6. Peter W. Jones, Ratios of interpolating Blaschke products, Pacific J. Math. 95 (1981), no. 2, 311-321. MR0632189(82m:30032)

7. Donald E. Marshall and Arne Stray, Interpolating Blaschke products, Pacific J. Math. 173 (1996), no. 2, 491-499. MR1394401 (97c:30042)

8. Kristian Seip, Interpolation and sampling in spaces of analytic functions, University Lecture Series 33, American Mathematical Society, Providence, RI, 2004. MR2040080

Department of Mathematical Sciences, Norwegian University of Science and TechNOLOGY, 7491 TRONDHEIM, NORWAY

E-mail address: hjelle@math.ntnu.no

URL: http://www.math.ntnu.no/ hjelle/ 